\title{
The concept of application of oscillation energy in modern technologies of directional drilling
}

\author{
Miroslava Chernova ${ }^{1}$, and Yaroslav Kuntsiak $^{1 *}$ \\ ${ }^{1}$ Ivano-Frankivsk National Technical University of Oil and Gas, Department of General and Applied \\ Physics, 15 Karpatska Ave., 76018, Ivano-Frankivsk, Ukraine
}

\begin{abstract}
In the process of research, there was investigated the question of influence of rotary movement of washing fluid on hydrodynamic processes with the purpose of enhancement of its transportation properties, especially in the process of drilling of horizontal wells. At present stage of science and technology development, improvement of effectiveness of drilling of horizontal wells is done at the expense of improvement of hydrodynamic circulation processes that prevent complications related to stability disturbances of well walls, formation of stagnation zones, and separation of sludge with significant reduction of energy costs. The matter of the article is related to the process of formation of stability of rotaryforward motion of washing fluid in the annulus of horizontal well directly by the energy of the flow, and also substantiation of methods of regulation of hydrodynamic parameters and flow structure with consideration of concrete geological and technical conditions.
\end{abstract}

\section{Introduction}

According to the information project "Unconventional gas in Ukraine", most natural gases $\left(\mathrm{CH}_{4}, \mathrm{C}_{2} \mathrm{H}_{6}, \mathrm{C}_{3} \mathrm{H}_{8}, \mathrm{CO}_{2}, \mathrm{~N}_{2}, \mathrm{H}_{2} \mathrm{~S}\right.$, etc.) form hydrates that exist under certain thermobaric conditions [1]. However, the main prospects for their location are associated with depths of 400-1600 m from the seabed, the thickness of rocks in which the conditions of existence of gas-hydrate deposits are favorably, is from 170 to $480 \mathrm{~m}$ [2-5].

Currently, several variants of methane production technologies from gas hydrates have been developed [6-9]. The all most well-grown technologies for methane production from gas hydrates are:

- depressive method is to artificially reduce the pressure or depressurize the gas hydrate below equilibrium by pumping water from the well. The method of reducing the pressure is suitable for hydrate layers, where the saturation of hydrates is low [10]. The disadvantage of the method of reducing the pressure is associated with the formation of hydrates in the bottomhole zone, which complicates the process of gas production [11];

- thermal method is to heat the gas hydrate layer by injecting steam or hot water [12]. The use of thermal methods is justified only if the total energy consumption for the decomposition of hydrates in the formation does not exceed the amount of energy that can

${ }^{*}$ Corresponding author: miracherri1@,gmail.com 
be obtained from the extracted gas [3, 14-16]. The thermal method of developing gas hydrate fields can be applied to formations with a high content of hydrates;

- substitution technology is the displacement of methane from the "cells" of clarates by filling them with another gas, such as carbon dioxide. This technology has been developed recently $[5,12,17]$.

The essence of the technology of production with the replacement of carbon dioxide is that carbon dioxide is fed into the gas hydrate stratum $[18,19]$. In the structure of the gas hydrate, methane molecules are replaced by carbon dioxide molecules. The most attractive from an economic and technical point of view is the extraction of methane from methanohydrates by reducing the pressure $[10,20]$, it is possible to use the method of reducing the pressure together with periodic local heating of the volume near the well in the layer containing methanohydrates [17, 21-23].

Mykhailo Honchar, President of the Strategy XXI Center for Global Studies, noted that the practical implementation of any of the extraction projects involves a number of technological difficulties and risks, including environmental and technical ones, one of which is underwater drilling at great depths [19, 24-27]. Therefore, in the process of research we considered the influence of the rotational motion of the flushing fluid on the hydrodynamic processes to improve its transport properties, especially in the process of drilling deep wells [11]. At the present stage of development of science and technology to increase the efficiency of drilling deep wells is due to improved hydrodynamic circulation processes that prevent complications associated with violation of the stability of well walls, stagnation, sludge separation with a significant reduction in energy consumption. The essence of the work is to form the stability of the rotational motion of the flushing fluid in the annular channel of the directed deep well directly by the flow energy, as well as substantiation of methods for regulating hydrodynamic parameters and flow structure taking into account specific geological and technical conditions of underwater drilling.

Under the conditions of drilling deep wells for oil and gas, hydrates can be formed in these wellbore, deposited on the walls of drill pipes, causing corrosion-fatigue destruction of drill string elements. To combat this phenomenon, various inhibitors are added to the wells [28].

Of scientific interest are works where vibrational thermal convection is studied, which characterizes the influence of vibrational processes on the dynamics of inhomogeneous density of incompressible fluid. The behavior of different phase impurities (solid, droplet, gaseous) in a liquid exposed to forced oscillations has certain features. Among these features are two significant - the processes of vibration and the effects of localization, i.e. the directed movement of impurities (drilled rock) under the influence of oscillations and the formation in certain places of the liquid column of their local accumulations.

Theoretical studies show that the dynamics of phase components (solid or gaseous) in a liquid under the influence of periodic factors depends on the generation frequency. In the high frequency range, where the oscillation frequency is quite high, the effects of compression are not manifested, so when considering the oscillating motion of the fluid, the viscous near-wall stratum Stokes can be neglected and the outstream can be considered potential.

The oil and gas industry deals with viscous liquids, so studies of the effect of the oscillating process on liquids when their compressibility is insignificant are of practical interest. Of great importance are studies of the generation of oscillations for phase inclusions in liquids within moderate $\left(\sim 10^{2} \mathrm{~Hz}\right)$ and low $(\sim 1-10 \mathrm{~Hz})$ frequencies.

Studies have shown that solid impurities in the fluid during translation-oscillating motion under the action of inertial forces oscillate, and the hydrodynamic interaction of the body, which oscillates with the liquid, leads of the medium-force in time acting on this body. Since the oscillations of a fluid are anharmonic - the fluid has a different velocity in different directions (there is a gradient of the velocity of the fluid) - so the solid particles move up in the liquid column. 
The phenomenon of oscillatory motion of bodies can occur in asymmetric systems, and this asymmetry can be force, kinematic, structural, gradient, wave, and others. The movement of bodies is also possible under the influence of symmetrical oscillations of the mechanical system. Under the action of harmonic oscillations, impurities that have a higher density than liquid can emerge (Chelomey effect) [29]. Of particular interest are combined oscillations, in particular translational-rotational, when the rotating component causes oscillations of the fluid relative to the tubular or annular space, and the intense translational component causes synchronous oscillations of the solid fraction in the liquid, which are much larger than translational vibrations.

Studies of the effect of generating oscillations of average $\left(\sim 10-10^{2} \mathrm{~Hz}\right)$ frequencies acting on the body in a cavity with a liquid under the influence of combined oscillations have established that during oscillations the average force directed along the kinetic energy gradient of the oscillatory movements in the fluid.

Theoretical studies on the interaction of solids under the influence of oscillatory processes have shown that regardless of whether the oscillatory motion occurs in a liquid where there are solids, or the oscillatory motion occurs with a solid body in a liquid that does not undergo an oscillatory process, there is a hydrodynamic interaction bodies resulting the bodies certain of the parameters of were attracted. The force of attraction decreases rapidly with increasing distance between bodies, and acts only at distances commensurate with the size of the bodies themselves. It is established that the lifting and suspension of a heavy solid in a liquid by means of oscillations of another solid body above it does not depend on the viscosity of the liquid. This process is characterized by hysteresis, i.e. the adhesion of the lower body to the oscillating upper, and the fall of the lower body occurs at different parameters of oscillations [28].

Faraday once studied the behavior of a set of small particles on a vibrating surface. He studied the thin layers of the bulk medium in gases under the action of oscillations, and even then obtained results that were manifested in the movement of the sand mass and the emergence of geometrically ordered structures. According to the results of the study of bulk media in liquids under the influence of different types of oscillations, it was found that during the circular translational oscillations simultaneously with the appearance of relief on the surface of the bulk medium in the form of three-dimensional hexagonal structures, rotation of the whole sand layer was observed. In the case of rotational oscillations there is a movement of the bulk medium in the direction of the axis of oscillation. During the oscillation of the cavity itself, the surface of the sand is inclined.

Studies of sedimentation of the bulk medium in both vertical and inclined channels filled with liquid, carrying out translational longitudinal oscillations, found an interesting effect of stabilizing the lower limit of sand blocks, and the sedimentation rate in this is much lower than in the absence of oscillations.

\section{Mathematical representation and description of the dynamics of flow oscillations structure in the annulus in the well}

The dynamics of bulk medium in a liquid of a position of the influence of oscillations, the direction of which is tangent to the interface, showed the excitation of a quasi-stationary spatial periodic relief at the interface this mediums. As it turned out, the formation of the relief occurs after the transition of the bulk medium to a liquefied state and is associated with the manifestation of instability of tangential gaps at the interface of moving media relative to each other (Kelvin-Helmholtz instability). At this the behavior of the "sandliquid" system is similar to the behavior of two immiscible liquids with different densities during horizontal vibrational processes. To describe the dynamics of such a system, an oscillating analogue of the Fruud number was introduced for the first time: 


$$
W=\frac{2 b Q}{g L},
$$

where $b$-the amplitude of oscillations, $\mathrm{m} ; Q$ - cyclic frequency, $\mathrm{c}^{1} ; L$ - characteristic size, $\mathrm{m}$.

Theoretical studies of the dynamics of weakly inhomogeneous suspended solid phase in a liquid under the influence of high-frequency oscillations, in the case when the period of oscillations, relaxation time of particle velocity and liquid phase are proportional, showed the phase shift between the oscillations particls and liquids. It was found that during high interfacial friction and a frequency of $\sim 10^{2} \mathrm{~Hz}$ oscillations, the substance in the suspended state can be described within a single-liquid model, which takes into account the inertial properties of the liquid and particles in the suspended state and the effect of the accedence masses. It became obvious that along with the monotonic mechanism of excitation of instability there is a possibility of an oscillatory component. As it turned out, the range of moderate and low frequencies is not sufficiently studied [29].

The viscosity of the liquid plays a significant role in this frequency range. At distances corresponding to the thickness of the Stokes layer, repulsive forces are manifested, while at greater distances between the particles of the solid fraction, the forces of attraction dominate (Fig. 1). Studies of the lifting force in a wide range of oscillation frequencies have shown that in the low-frequency range $(1-10 \mathrm{~Hz})$ the forces of attraction between particles do not appear, and the repulsive forces decrease with the alienation of particles whis the law exponentionalitions.

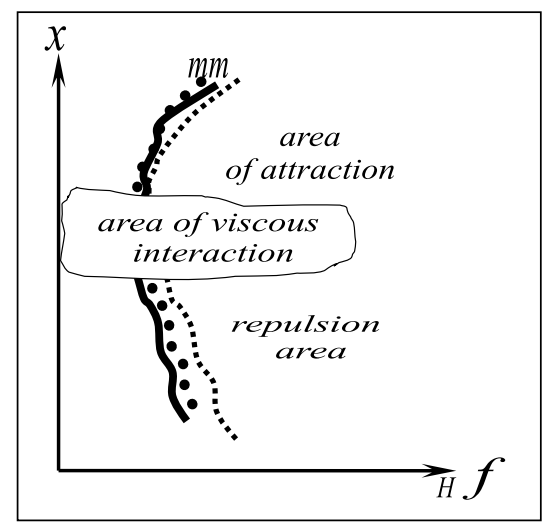

Fig. 1. Dependence of forces of attraction and repulsion on the distance between particles (difference between frequencies $30 \mathrm{~Hz}$ ).

In the case of high frequencies $\left(10^{2}-10^{3} \mathrm{~Hz}\right)$, the dependence of the interaction force on the distance between the body in the liquid and the wall has a minimum, and the repulsive force dominates at the boundary, which decreases rapidly with distance. Beyond the region of viscous interaction, the force of vibrational attraction is manifested, which also decreases with distance. Depending on the range of frequency oscillations, the heavy fraction of the drilled rock may be in a suspended state in a static force field.

The study of the behavior of a solid in a liquid under the influence of rotational oscillating motion as a function of frequency showed a nonmonotonic change in the parameter $W r$, which characterizes the rise and fall of bodies in a liquid in the gravitational field. It is established that in the range of moderate $\left(\sim 10^{2} \mathrm{~Hz}\right)$ and low $(\sim 1-10 \mathrm{~Hz})$ frequencies the effect of floating of solid particles of the drilled fraction is complicated by the presence of forces of close interaction with the walls the column of the drillings: abrupt transitions of the body from one boundary to another the intensity of oscillations is 
preceded by a monotonic movement of the particle from the boundary to a distance of the order of the viscous near-wall stratum Stokes layer. Thus, the dynamics of the solid fraction of drilled rock in a viscous fluid during the rotational oscillations of the drill string is determined by two phenomena. One of them is the phenomenon of levitation of a heavy body in a pulsating moving flow, which is not associated with the presence of the walls of the drill string and is determined only by the heterogeneity of the flow. Of second is the phenomenon of close viscous interaction with the walls of the drill string, which is manifested at distances of the order of the thickness of the Stokes layer. The first phenomenon decreases with increasing viscosity of the liquid, the vibrational lifting force decreases, and the second increases with increasing thickness of the Stokes viscous layer.

Since there is a certain hydrodynamic interaction between the particles of the drilled rock in the fluid flow, it is of great interest to study the influences of oscillatory processes on this interaction.

Studies of the effect of rotational oscillations on the close hydrodynamic interaction of particles have shown that in low-viscosity fluids, mostly is the repulsively of the interaction. The repulsive force decreases rapidly with distance. In the range of high frequencies of oscillations during the interaction of bodies, the amplitude of the velocity of oscillations plays a significant role. In viscous liquids, as the frequency decreases, the repulsive forces weaken, there is a significant change in the nature of the vibrational interaction at relatively short distances, namely, the particles begin to be attracted.

The study of the dynamics of the bulk medium, which consists of a large number of particles in an incompressible viscous fluid under the action of horizontal translational oscillations, showed that under the influence of the oscillations of tangents to the boundary the media, the component of quick sanding becomes unstablesion, with the transition to a liquefied state. The relief that develops is in the form of a stable system of ridges oriented perpendicular to the axis of oscillation. As the intensity of oscillations decreases, the dynamic relief disappears, and the bulk medium turns into a quasi-solid state. With decreasing frequency, the excitation threshold of the dynamic relief increases, and hysteresis appears in the transitions of the bulk medium to the liquefied and quasi-solid states. Studies have been performed for different variations in particle size and viscosity of liquids.

The supercritical dynamics of the relief depending on the parameters of oscillations and the characteristic particle sizes of the bulk medium are studied. It is established that in the case of small particles the change of the characteristic relief period is nonmonotonic, which is explained by the processes of liquefaction of the bulk medium. Structures with doubled spatial period are revealed. It is possible to form a stable dynamic system of twodimensional ridges separated by a clear liquid, with almost vertical slopes. In experimental and theoretical results, the liquefied bulk medium and the pure liquid were considered as two immiscible liquids, and at the interface of the surface tension force are absent.

The fluid moving through the annulus in the process of drilling vertical, inclined and horizontal wells is a jet turbulent flow, in different parts of which there are both free and near-wall shear layers with coherent structures that develop under the action of vibrationoscillating processes. In such structures there are: critical points with maximum heat and mass transfer coefficients; sharp curvature of flow lines; in the parietal part of the jet there are local non-stationary flow separations. Therefore, the study of fundamental physical effects and accompanying phenomena is relevant.

In jet streams, the existing coherent structures are relatively large-scale deterministic vortices in the mixing layers, which are characterized by a significant lifetime. These structures result from the fusion of smaller vortices that develop in the shear layer as a result of Kelvin-Helmholtz instability. Coherent structures contain a significant part of the turbulent kinetic energy of the flow and largely determine the intensity of the transfer processes. 
It is well known that external periodic perturbations of the flow affect the characteristics of the jet. Excitation of the flow at the directional frequency of coherent structures, as a rule, leads to their regularization and amplification. Thus, the influence of even lowamplitude (low-energy) perturbations on the flow makes it possible to influence the turbulent characteristics and heat and mass transfer processes in the flow [29] (Fig. 2).

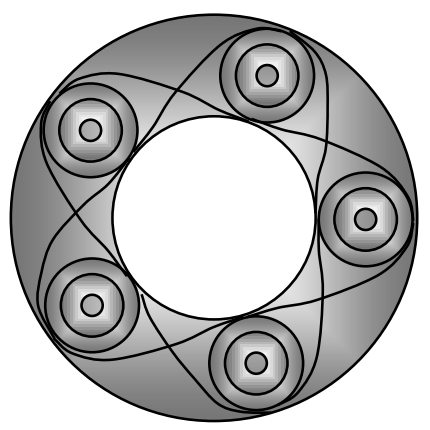

Fig. 2. Block diagram of instantaneous node locations.

The complex potential of the fluid flow with intensity $\chi$, the center of turbulence of which is at some point $z_{0}$, is described by the equation:

$$
E F=\omega=i \chi \ln \left(z-z_{0}\right) .
$$

The resulting structure in the cross section around the pipe in the annulus under the action of oscillations is shown in Fig. 2, where the lines connect the instantaneous locations of the nodes.

The general equation of fluid motion in the structure can be represented as:

$$
\frac{d x}{d t}=\frac{\partial \Psi(x, y, t)}{\partial y} ; \frac{d y}{d t}=-\frac{\partial \Psi(x, y, t)}{\partial y},
$$

and the equations that describe the flow lines, taking into account the structure that arises under these conditions, has the form:

$$
\Psi=\frac{\chi}{4 \pi} \ln \left((x \pm 2 a)^{2}+y^{2}\right)+\frac{\chi}{4 \pi} \ln \left(x^{2}+y^{2}\right)+\frac{\chi}{4 \pi} \ln \left((x \pm 2 a)^{2}+y^{2}\right)-\frac{25 \chi}{96 \pi} \ln \left(x^{2}+y^{2}\right),
$$

where $x$ and $y$ - are the coordinates of the axes in the cross section of the fluid flow; $a$ - the distance between the flow layers in the radial direction; $\chi$ - flow intensity.

Then the complex motion potential of the fluid of such a system is described by an equation having the form:

$$
\omega_{n}=i \chi \ln (z)+i \chi \ln (z \pm a)+\ldots+i \chi(z \pm n a) .
$$

Based on the results of studies of the patterns of development of large-scale structures in close proximity to solid surfaces, which in our case may be the wellbore wall or drill string surface, we considered the possibility of flow control by creating periodic oscillations to effectively influence the intensity of transfer process in limited jet currents, which are mobile washing fluids in the annulus of the well [28].

Experimental studies concerned the study of the critical point around dual electro diffusion friction sensors and the Particle Image Velocimetry (PIV) method. The PIV 
method is based on the laws of statistical physics, which has recently become quite widely used. The Gibbs distribution, the Monte Carlo method, and others are most often used.

This made it possible to determine the characteristics of zones of nonstationary local separations and on the basis of the ability to control the spectrum of turbulent pulsations using various methods of active influence, to investigate the sensitivity of the jet to external periodic perturbations of different frequency and amplitudes. It was shown for the first time that low-amplitude external perturbation of the jet at frequencies in the range of maximum susceptibility leads to a decrease in the magnitude of the friction force and a sharp increase in the level of pulsations. It is established that the resonant growth of pulsation oscillations of the jet is closely related to the coherent component.

Studies have shown the effect of attenuation of coherent structures in liquids with a fine gas phase. The range of possible flow regimes was determined - from self-oscillations to stationary ones with stable jet connection. The hysteresis phenomena during the continuous change of the boundary conditions for the leakage of jets into a limited space, which in our case is the annulus of the well, are investigated. A significant influence of the Coanda effect on the regularities of jet flows in the annulus of the well has been revealed.

In the course of research the influence of rotational movement of the washing liquid on hydrodynamic processes for increase of its transporting properties, especially in the course of drilling of horizontal wells was considered. We [29] showed the increase of drilling efficiency of horizontally directed wells due to the improvement of hydrodynamic circulation processes, which prevent complications associated with the violation of the stability of the well walls, the formation of stagnant zones, sludge separation with a significant reduction in energy consumption. The content of the work is to form the stability of the rotational motion of the flushing fluid in the annular channel of the horizontal well directly by the flow energy, as well as substantiation of methods for regulating hydrodynamic parameters and flow structure according to the power law taking into account specific geological and technical conditions.

Based on mathematical modeling of hydrodynamics, filtration, heat and mass transfer, using a systematic approach, the possibility of analyzing the dynamics of flow and pressure in different parts of the system is proved, which allows to determine conditions and calculate the kinetics of mass transfer and filtration processes in wells and shafts, estimating bottomhole processing energy. This allows the selection of cost-effective modes during the layout of downhole equipment and the search for new technical solutions in the pulsation technology of cleaning the wellbore and bottomhole zone.

Mathematical modeling of hydrodynamics, filtration, heat and mass transfer, using a system approach, analyze the dynamics of flow and pressure in different parts of the system, determine the conditions and calculate the kinetics of mass transfer and filtration processes in the well and formation, estimate energy consumption in the wellbore zone.

In particular, during the calculations, the flow function is used $\psi$, which determines the velocity field from the condition:

$$
\begin{gathered}
u=\left(0, \frac{1}{\sin \theta} \frac{\partial \Psi}{\partial \varphi},-\frac{\partial \Psi}{\partial \theta}\right) \\
\Delta \Psi=-\omega=\frac{1}{\sin \theta} \frac{\partial}{\partial \theta}\left(\sin \theta \frac{\partial \Psi}{\partial \theta}\right)+\frac{1}{\sin ^{2} \theta} \frac{\partial^{2} \Psi}{\partial \varphi^{2}},
\end{gathered}
$$

where the Beltrami-Laplace operator is used in the right part, and to fulfill Kelvin's theorem, the Green's $G\left(\theta, \varphi, \theta^{\prime}, \varphi^{\prime}\right)$ function is used for circulation, then the BeltramiLaplace operator is used in the form: 


$$
G\left(\theta, \varphi, \theta^{\prime}, \varphi^{\prime}\right)=-\frac{1}{2 \pi} \ln \sin \left(\frac{1}{2} \gamma\left(\theta, \varphi, \theta^{\prime}, \varphi^{\prime}\right)\right),
$$

where $\gamma\left(\theta, \varphi, \theta^{\prime}, \varphi^{\prime}\right)$ - is the central angle between the points with coordinates $(\theta, \varphi)$ and $G\left(\theta^{\prime}, \varphi^{\prime}\right)$.

By applying the Green's function and the formulas of trigonometric transformations, the flow function $\psi$ will take the form:

$$
\Psi(\theta, \varphi)=\int_{S} \omega\left(\theta^{\prime}, \varphi^{\prime}\right) \ln (1-\cos \gamma) \sin \theta^{\prime} d \theta^{\prime} d \varphi^{\prime},
$$

where the integration is carried out over the area of the cross-sectional ring.

For the $N$-th number of perturbations, the equations of motion are written in the form:

$$
\begin{gathered}
\dot{\theta}_{i}=-\frac{1}{4 \pi} \sum_{j=1, i \neq j}^{N} \Gamma_{j} \frac{\sin \theta_{j} \sin \left(\varphi_{i}-\varphi_{j}\right)}{1-\cos \gamma_{i j}} ; \\
\sin \theta_{i} \varphi_{i}=-\frac{1}{4 \pi} \sum_{j=1, i \neq j}^{N} \Gamma_{j} \frac{\cos \theta_{i} \sin \theta_{j} \cos \left(\varphi_{i}-\varphi_{j}\right)-\sin \theta_{i} \cos \theta_{j}}{1-\cos \gamma_{i j}},
\end{gathered}
$$

where $\gamma_{i j}=\gamma\left(\theta, \varphi_{i}, \theta_{j}, \varphi_{j}\right) ; \Gamma_{j}$ - intensity of the $j$-th perturbation.

The process of describing hydrodynamics and nonstationary filtration takes into account the relationship between the individual parts of the pulsating system based on the analysis of a multicomponent nonlinear system of differential equations.

Studies [28] show that low-frequency pulsations accelerate the processes of relaxation of mechanical stresses by two or three orders of magnitude, which eliminates the negative effects of drilling and perforation shots, helps maintain formation pressure and its uniformity within the formation. This fact is common to all pulsating hydrodynamic modes of drainage during cleaning of the wellbore and bottomhole zone.

Analysis of the results of industrial research on oil wells of domestic and foreign companies confirms the constant regularity of processes occurring under the influence of pulsation treatment, namely: gas yield, release of viscous oil-water emulsion and liquid with oil film, removal to the surface of the light mechanical impurities in the form of salts and oxides.

Studies of the motion of solid inclusions of a liquid in an inhomogeneous standing wave have shown that particles can be localized in antinodes and in wave nodes both on the wall surface and at a certain distance from it in the form of rings. It is established that depending on the type of oscillation source, the frequency of oscillations of solid particles can be concentrated not only in the longitudinal direction, but also in the transverse direction. The combined effect on the mixture of both ultrasound and low-frequency oscillations was also considered. The results of research have shown that with the help of low-frequency oscillations it is possible to force local clusters to move in the vertical direction.

To study the stability of relative equilibrium positions, the criterion formulated is that if the Hamiltonian in the coordinate system moving with the system reaches its transverse minimum or maximum in the equilibrium position, then such an equilibrium position is stable.

To describe such a system, it is successful to use Hamilton's equations with the following Hamiltonian and canonical variables:

$$
H=\frac{1}{4 \pi} \sum_{i<j} \Gamma_{i} \Gamma_{j} \ln \ell_{i j}
$$




$$
\begin{gathered}
P=\sqrt{\left|\Gamma_{i}\right|} \cos \theta_{i} ; Q=\sqrt{\left|\Gamma_{i}\right|} \varphi_{i} \\
\dot{P}_{i}=\frac{\partial H}{\partial Q_{i}} ; \dot{Q}=-\frac{\partial H}{\partial P_{i}},
\end{gathered}
$$

where $\ell_{i j}^{2}=\left(X_{i}-X_{j}\right)^{2}=2\left(1-\cos \gamma_{i j}\right)$.

The Poisson parenthesis for this Hamiltonian system has the form:

$$
\{f, g\}=\sum_{k=1}^{N} \frac{1}{\Gamma_{k}}\left(\frac{\partial f}{\partial \cos \theta_{k}} \frac{\partial g}{\partial \varphi_{k}}-\frac{\partial f}{\partial \varphi_{k}} \frac{\partial g}{\partial \cos \theta_{k}}\right) ;\left\{\varphi_{i}, \cos \theta_{j}\right\}=\frac{\delta_{i j}}{\Gamma_{i}}
$$

where $\delta_{i j}$ Kronecker symbol.

In addition to the Hamiltonian $H$, this system allows three more integrals of motion:

$$
\begin{gathered}
M_{1}=\sum_{i=1}^{N} \Gamma_{i} \sin \theta_{i} \cos \varphi_{i}=\text { const } \\
M_{2}=\sum_{i=1}^{N} \Gamma_{i} \sin \theta_{i} \sin \varphi_{i}=\text { const } \\
M_{3}=\sum_{i=1}^{N} \Gamma_{i} \cos \theta_{i}=\text { const }
\end{gathered}
$$

forming a vector called the moment of perturbation.

The above values $H, M_{1}, M_{2}, M_{3}$ give three independent involutive integrals:

$$
\begin{gathered}
\left\{H, M_{3}\right\}=0 ; \\
\left\{H, M_{1}^{2}+M_{2}^{2}\right\}=0 ; \\
\left\{M_{3}, M_{1}^{2}+M_{2}^{2}\right\}=0 .
\end{gathered}
$$

The position of equilibrium or relative equilibrium in the system, which in our case causes the retention of a particle in the middle of the liquid, is also described by equations in vector form, such as:

$$
\begin{aligned}
& \dot{X}_{i}=\sum_{k=1, k \neq i}^{N} \frac{\Gamma_{k}}{2 \pi} \frac{X_{k} \cdot X_{i}}{\left(X_{i}-X_{k}\right)^{2}}+\Omega e_{z} \cdot X_{i} ; \\
& \dot{X}_{j}=\sum_{k=1, k \neq j}^{N} \frac{\Gamma_{k}}{2 \pi} \frac{X_{k} \cdot X_{j}}{\left(X_{j}-X_{k}\right)^{2}}+\Omega e_{z} \cdot X_{j},
\end{aligned}
$$

and after mathematical transformations for $\ell_{i j}^{2}=\left(X_{i}-X_{j}\right)^{2}$ we receive:

$$
\frac{d \ell_{i j}^{2}}{d t}=\sum_{k=1, k \neq i, k \neq j}^{N} \Gamma_{k} \frac{V_{i j k}}{\pi}\left(\frac{1}{\ell_{i k}^{2}}-\frac{1}{\ell_{j k}^{2}}\right),
$$


where $V_{i j k}=X_{i} \cdot X_{j} \cdot X_{k}$.

The equilibrium position is present in the case when $\frac{d \ell_{i j}^{2}}{d t}=0$. Then the equation is written in matrix form, and their solution makes it possible to determine the matrices in the kernels of which there is a predetermined vector $\Gamma$. Then it is determined which of the found matrices contain a structure given by the equilibrium relation. On the other hand, for a given matrix we can find a limited number of intensities $\Gamma$ for a given flow, taking into account all its rheological parameters, at which the system (12) is in equilibrium, and then the problem is reduced the description of the kernel of a linear operator. Thus, there are symmetrical equilibrium positions for dispersed particles in the flow of washing liquid under the influence of vibrational forces.

The above theoretical, experimental studies and mathematical calculations with the choice of certain mathematical models make it possible to calculate the parameters of the fluid (pressure, velocity), design characteristics of devices that could ensure the presence of oscillating processes of a certain frequency, amplitude, energies to reduce wellbore the energy consumption and increasing the drilling speed of inclined and horizontal wells.

Based on the results of calculations and experience of experimental and industrial research, we have developed a series of hydrodynamic pulse generators to influence the oscillating processes to improve the efficiency of drilling deep directional wells.

Depending on the distribution of the axial velocity at the inlet to the generator, the process of formation of the power law of torsion of the flow and the physico-rheological properties of the washing liquid, the define a steady rotational-translational motion of the flow in the annular channel of pipe is set. With certain properties, the flow has favorable conditions for regulating the values of differential pressure, which depends on specific geological and technical conditions.

Steady rotational movement of the flow is achieved at certain values of the Fruud number $(F r)$, which are different for liquids with different rheological properties, and for the Reynolds number $(R e)$ the Newtonian fluids must have a value twice as large as the non-Newtonians fluids; and there is a functional connection between them according to the parabolic law.

The rotational movement of the flow in the annular horizontal channel at the inlet undergoes an axial velocity distribution, and at the outlet conditions are created in which the circular component of the velocity is greater than the axial power distribution. In this case, the inertial forces exceed the viscosity forces. The specific circular flow rate of the washing liquid is used as a control parameter.

In combination with the layout of the bottom of the drill string hydrodynamic generators provide increased drilling speed (reduction of drilling time by $25-40 \%$ ), better cleaning of the wellbore in both inclined and horizontal wells due to physical wave processes that provide prolonged stay of the solid fraction in a suspended state and its directed movement in the direction of removal to the day surface. At the same time, the process of sticking of the small fraction on the casing is impossible. It is also important that the use of our developed devices with certain technological parameters provides the formation of a colmatation layer of small thickness.

The use of the hydrodynamic devices developed by us makes it possible to control the technological processes of drilling inclined and horizontal wells with downhole engines and to optimize the process of underwater deep drilling in specific geological conditions.

At the same time, oscillatory processes in the low-frequency range, which occur under the conditions of using our proposed hydrodynamic devices, prevent gas hydrate formations on the walls of the elements of the drill string. 


\section{Conclusions}

1. Pre-calculating the range of oscillations generated at the bottom of the well using devices placed in the bottom of the drill string, and taking into account the rheological properties of the flushing fluid for these geological and technological conditions, which can be changed under the influence of the same generated oscillating processes:

- to increase the efficiency of cleaning the well from the drilled rock due to those physical processes and phenomena that occur under the action of fluctuations in the fluid, regardless of the depth of the well;

- reduce the thickness of the colmatation layer of the wellbore;

- to ensure the absence of adhesion of the smallest particles of drilled rock on the body surface of the drill string;

- significantly reduce the likelihood of jamming the drill string during drilling of deep wells, preventing stagnation.

2. Taking into account the above possibilities, the interval of reduction of time and energy consumption for the construction of the above-mentioned wells is up to $30-40 \%$ of the usual.

3. Empirical selection of device parameters that become a source of oscillating processes of the required frequency in the well is impossible.

4. Oscillatory processes of the low-frequency range are characterized by a significant impact on gas hydrate formations.

This work was conducted within the projects "Scientific basics to form unified system to preserve and generate power of the objects of fuel and energy complex of Ukraine" (State registration No. 0117U001127) and "Scientific substantiation and development of energy saving and low waste technologies of hydrocarbon and mineral raw materials extraction" (State registration No. 0116U008041). The authors of the article express their sincere gratitude to the employees of "Research Institute of Drilling Instrument Design Bureau" for consulting support and assistance in conducting industrial tests.

\section{References}

1. Vagnetti, R., Boswell, R., \& Pratt, S. (2013) Researching the climate change implications of methane hydrates. Environment Coastal \& Offshore (ECO), 28-33.

2. Shnyukov, Y.F. (2015). Treasures of the Black Sea. Conversation with geologist, academician of the National Academy of Sciences of Ukraine. Retrieved from http://www.vokrugsveta.com/S4/nature/blacksea.htm

3. Remer, M., Saling, H., Bar, A., Feseker, T., Bintersteler, P., \& Borman, G. (2012). Heolohichnyi kontrol i velychyna ebulitsii metanu z raionu bysokyh potokiv u Chornomu mori - raiony Kerchenskoho prosochuvannia. Morska Heolohiia, (319-322), 57-74.

4. Gonchar, M. (2013). Tretia hazova revoliutsiia. Retrieved from http:/tyzhden.ua/Economics/75194

5. Zarubin, Jy.O., Gunda, M.V., Gryshanenko, V.P., Burenkov, V.V., \& Shvydkyj, O.A. (2012). Rozrobka morskyh rodovysth nafty i gazu. DP «Naukanaftogaz» Natshionalnoji aktsionernoji kompaniji «Naftogaz Ukrajiny», (312).

6. Volovich, O. (2016). Stan i perspektyvy osvoyennia vydobutku gazogidrativ v ukrayinskomu sektori Chornogho moria. Analitychna Zapuska.

7. Li, X.-S., Yang, B., Li, G., Li, B., Zhang, Y., \& Chen, Z.-Y. (2012). Experimental study on gas production from methane hydrate in porous media by huff and puff method in Pilot-Scale Hydrate Simulator. Fuel, (94), 486-494. https://doi.org/10.1016/j.fuel.2011.11.011

8. Aminnaji, M., Anderson, R., \& Tohidi, B. (2019). Anomalous KHI-Induced dissociation of gas hydrates inside the hydrate stability zone: Experimental observations \& potential mechanisms. 
Journal of Petroleum Science and Engineering, (17)8, 1044-1050. https://doi.org/10.1016/j.petrol.2019.03.086

9. Makohon, Yu. F. (2003) Prirodnyie gazovyie gidraty: raspoodgeniye, modeli obrazovaniya, resursy. Gurnal Rossijskogo Himicheskogo Obshestva im. D.I. Mendeleyeva, XLVII(3), 70-79.

10. Gas Hydrates JIP. (n.d.) Retrieved from http://gomhydratejip.ucsd.edu/

11. Su, Zh., Moridis, G.J., Zhang, K., \& Wu, N. (2012) A huff-and-puff production of gas hydrate deposits in Shenhu area of South China Sea through a vertical well. Journal of Petroleum Science and Engineering, (86-87), 54-61.

12. Bondarenko, V., Ganushevych, K., Sai, K., \& Tyshchenko, A. (2011). Development of gas hydrates in the Black sea. Technical and Geoinformational Systems in Mining, 55-59. https://doi.org/10.1201/b11586-11

13. U.S.A and Japan Complete Successful Field Trial of Methane Hydrate Production Technologies. (2012). Department of Energy, USA, May 2, 2012. Retrieved from http://energy.gov/articles/usand-japan-complete-successful-field-trial-methane-hydrate-production-technologies

14. Natural Gas Flow Test from Methane Hydrate Layers Ended. (2013). Agency for Natural Resources and Energy of Ministry of Economy, Trade and Industry, Japan, March 18, 2013. Retrieved from http://www.meti.go.jp/english/press/2013/0318_03.html

15. Japan's Methane Hydrate R\&D Program. (2018). Phase 1 Comprehensive Report of Research Results. Research Consortium for Methane Hydrate Resources in Japan, (69).

16. Submarine Gas Hydrate Reservoirs. SUGAR project. (2013). GEOMAR Helmholtz Centre for Ocean Research Kiel. Retrieved from http://www.geomar.de/en/research/fb2/fb2mg/projects/sugar-i/

17. Sokur, O.N. (2010). Mirovoj opyt podhoda k reshenijy probliemy ispolzovanija gazogidratov kak istochnika elektroenergeticheskogo syrja. Zbirnyk Naukovyh Prats Instytutu Heolohichnyh Nauk NAN Ukrainy, (3), 343-349.

18. Methane Hydrates and the Potential Natural Gas. (2013). Boom Natural Gas Europe. Retrieved from http://www.naturalgaseurope.com/methane-hydrates-and-the-potential-natural-gas-boom

19. Kobolev, V. (2010). Doslidno-metodychna kompleksna heoloho-heofizychna ekspedytsiia 66-ho reisu NDS “Profesor Vodianytskyi” v zahidnii chastynii Chornoho moria. Geolog Ukrainy, (4), 40-62.

20. Gonchar, M. (2013). Hazohidraty Chornohgo moria: Investytsiia v enerhetychne maibutnie YeS ta Ukrainy. Retrieved from https://nomos.com.ua/content/view/427/86

21. Sokrovischa Chornogo moria. Beseda s geologom, akademikom NAN Ukrainy Yevgeniyem Fedorovichem Shniukovym. Retrieved from http://www.vokrugsveta.com/S4/nature/blacksea.htm

22. Shniukov, Ye.F., Pasynkov, A.A., \& Menasova, A.Sh. (2011) Projavlrnija masshtabnoj degazatsiji na akvatorijah Chornogo i Azovskogo мorej. Mineralni Resursy Ukrainy,(4), 35-38.

23. Makogon, Yu.F. (2010) Gazogidraty. Istorija izuchenija i perspektivy osvojenija. Geologija $i$ Poleznyje Iskopajemyje Mirovogo okeana, (2), 5-21.

24. Jegorov, V.M., Artemenkov, Ju.G., Gulin, S.B., \& Polikarpov, G.G. (2011) Metanovi sypy u Chornomu mori seredoutvoriujucha $j$ ekologichna rol. Sevastopol: NVTs ECOSIGIDROFIZYKA.

25. Donchenko, S.I. (2009). Gidratoakusticheskije priznaki gazogidratov i vozmogjnosti ih uchota pri modelirovanii sredy. Gidroakustychnyj Gurnal, (6), 36-51.

26. Kevin Biddl. (n.d.). V Ukraine jestho nie bylo projektov po glubokovodnomu bureniju takih masshtabov, kak nash. Retrieved from http://www.kommersant.ua/doc/2231705

27. Komatsu, H., Ota, M., Smith, R.L.Jr., \& Inomata, H. (2013). Ogliad laboratornyh doslidjen reaktsij zamishtshennija gidrativ klatratu $\mathrm{CO}_{2}-\mathrm{CH}_{4}-$ vlastyvosti ta kinetyka. Zhurnal Taivanskoho Instytutu Himichnyh Inzheneriv, (44), 517-537.

28. Chernova, M. (2019) Scientific trends in drilling deep oil and gas wells. International Academy Journal Web of Scholar, 1(31), 18-39. https://doi.org/10.31435/rsglobal_wos/31012019/6308/

29. Chernova, M.Ye. (2016). Dejaki metody teoretychny ta eksperymentalnyh doslidghen $v$ burinni. Nauchnyje Otvety na Vyzov Sovremennosti: Tehnika i Tehnologii, (1), 76-107. 